\section{Causas externas entre menores de \\ 15 anos em cidade do Sul do \\ Brasil: atendimentos em pronto- \\ socorro, internações e óbitos}

\section{External causes among individuals under 15 years of age in a city in south Brazil: emergency care, hospitalizations and deaths}

\section{Christine Baccarat de Godoy Martins ${ }^{1 *}$}

\section{Selma Maffei de Andrade ${ }^{2}$}

${ }^{1}$ Mestre em Saúde Coletiva pela Universidade Estadual de Londrina. Universidade Estadual de Londrina. Centro de Ciências da Saúde. Departamento de Enfermagem. Área: Saúde da Criança e do Adolescente.

${ }^{2}$ Doutora em Saúde Pública pela Faculdade de Saúde Pública - Universidade de São Paulo. Universidade Estadual de Londrina. Centro de Ciências da Saúde. Departamento de Saúde Coletiva.

\section{Resumo}

Objetivo: Identificar causas de atendimento hospitalar ou de morte e lesões entre menores de 15 anos vítimas de causas externas (acidentes ou violências), residentes em Londrina, Paraná, em 2001. Método: Estudo transversal, com coleta retrospectiva de dados. As informações sobre morbidade foram coletadas em prontuários dos cinco hospitais gerais da cidade, e as de mortalidade no Núcleo de Informação em Mortalidade do Município, com classificação dessas causas e do trauma gerado de acordo com a Classificação Internacional de Doenças. Resultados: Foram estudados 8.854 menores de 15 anos, com 95,7\% de atendimento em pronto-socorro e alta. Entre os atendidos em pronto-socorro, com alta, houve predominância de quedas (33,9\%), seguidas de eventos de intenção indeterminada $(31,8 \%)$ e de acidentes causados por forças inanimadas (15,5\%), principalmente por penetração de corpo estranho em orifício natural $(4,6 \%)$ ou através da pele $(3,2 \%)$. Nesse nível de atenção predominaram traumatismos superficiais $(32,9 \%)$ e ferimentos (29,3\%). Quedas também foram as principais causas entre os internados (32,4\%), seguidas de acidentes de transporte (19,5\%), acidentes causados por forças inanimadas $(15,7 \%)$ e envenenamentos $(13,5 \%)$. Entre os internados, as principais lesões foram traumatismos superficiais $(22,0 \%)$ e fraturas $(19,5 \%)$. Acidentes de transporte $(44,4 \%)$ e afogamento $(16,7 \%)$ foram as principais causas externas de óbito e o traumatismo crânio-encefálico a principal lesão fatal (50,0\%). Conclusões: As causas e os tipos de lesão diferem segundo o nível de atenção e indicam a necessidade de reestruturação dos serviços de saúde visando descentralizar o atendimento às lesões de menor complexidade.

Palavras-chave: Criança. Adolescente. Acidentes. Violência. Atendimento de emergência. Morbidade. Mortalidade. 


\section{Abstract}

Objective: To identify causes of hospital attendance or deaths due to external causes of morbidity and mortality (accidents or violence) and types of injury among children under the age of 15 who lived in Londrina, Paraná, Brazil, in 2001. Methods: Cross-sectional study, with retrospective data collection. Medical records of the five general hospitals and the Mortality Information Centre of the city were the source of morbidity and mortality data, respectively. External causes and types of injury were classified according to the International Classification of Diseases. Results: Out of 8,854 children, $95.7 \%$ received care in emergency rooms and were discharged. Among victims seen in emergency rooms, falls were the main type of accident (33.9\%), followed by events of undetermined intent (31.8\%), and accidents caused by inanimate forces (15.5\%), especially by foreign bodies in natural orifices (4.6\%) or through the skin (3.2\%). At this level of care, superficial trauma (32.9\%) and open wounds $(29.3 \%)$ were the main types of injury. Among children who were admitted to hospitals, falls were also the main type of accident (32.4\%), followed by transportation accidents (19.5\%), accidents caused by inanimate forces $(15.7 \%)$, and poisoning (13.5\%). Superficial trauma (22.0\%) and fractures (19.5\%) prevailed among admitted children. Transportation accidents $(44.4 \%)$ and drowning (16.7\%) were the main external causes of death and intracranial trauma (50.0\%) the main type of fatal injury. Conclusions: The causes and types of injury differ according to the level of care and indicate the need of restructuring healthcare services in order to decentralize care to less complex injuries.

Key Words: Child. Adolescent. Accidents. Violence. Emergency medical services. Morbidity. Mortality.

\section{Introdução}

Os acidentes e violências, denominados pela Classificação Internacional de Doenças, décima revisão, como causas externas de lesões e envenenamentos ${ }^{1}$, têm sido causas constantes de atendimentos e de internações no Brasil, resultando em alta demanda aos serviços de saúde e em sofrimento para as vítimas e seus familiares, além de elevados custos diretos e indiretos e de seqüelas, que comprometem a qualidade de vida dos que sofreram esses eventos ${ }^{2-6}$.

A criança, por sua imaturidade, curiosidade e intenso crescimento e desenvolvimento, encontra-se muitas vezes propensa a acidentes, e indefesa e vulnerável a violências. Somando-se a esses fatores, a alta incidência de causas externas em crianças e adolescentes tem despertado em todo o mundo a necessidade de estudos desses eventos na população infanto-juvenil ${ }^{6,7-14}$.

Conhecer o perfil de morbi-mortalidade infantil por causas externas possibilita, aos planejadores e executores de políticas públicas, definir em bases concretas as ações que deveriam ser prioritárias a fim de contemplar a prevenção e a atenção às vítimas dessas causas ${ }^{15}$. Estudar as causas e as conseqüências desse agravo, dessa forma, é essencial a fim de se formar um diagnóstico e contribuir para a adoção de medidas de prevenção, controle e assistência.

A presente investigação teve o objetivo de analisar as causas externas de atendimento em serviços de emergência, de internação e de óbito, e suas respectivas lesões, entre menores de 15 anos, residentes em Londrina, Paraná, e que foram vítimas de acidentes ou violências no ano de 2001.

\section{Método}

A pesquisa constituiu um estudo transversal, com coleta retrospectiva de dados. A população do estudo foi composta de menores de 15 anos de idade residentes no município de Londrina, Paraná, vítimas de acidente ou violência, atendidos em serviços hospitalares de urgência/emergência no período de 
1 de janeiro a 31 de dezembro de 2001, ou que morreram sem assistência médica por eventos ocorridos nesse ano ou em um prazo de até um ano após o acidente ou ato violento ocorrido em 2001, conforme preconiza a Organização Mundial de Saúde ${ }^{1}$.

Londrina situa-se no norte do estado do Paraná, com uma população projetada, para 2001, de 454.870 habitantes e uma taxa de urbanização de $97 \%$. Aproximadamente um quarto desses habitantes (26\%) é composto por crianças de zero a quatorze anos ${ }^{16}$.

Os dados de morbidade (atendimentos de emergência/urgência e internação) foram obtidos por meio de investigação em todos os hospitais gerais do município (cinco) que prestam atendimento a menores de 15 anos, sendo revistas todas as fichas de atendimento de emergência/urgência e prontuários de menores de 15 anos residentes em Londrina e selecionados os referentes a atendimento por causas não naturais (trauma, ferimento, envenenamento ou queimadura) no ano de 2001. Um formulário previamente testado foi utilizado para transcrição dos dados.

As informações sobre mortalidade foram obtidas no Núcleo de Informação em Mortalidade (NIM) da Prefeitura de Londrina, considerando que o óbito poderia ter ocorrido sem que o atendimento houvesse sido prestado por serviço de saúde. Esse Núcleo realiza investigação rotineira dos casos de morte por causas mal definidas ou por causas externas de intenção indeterminada, o que garante a boa qualidade dos dados. Além dos óbitos ocorridos por causas externas em 2001, também foram levantados os óbitos ocorridos pela mesma causa em 2002 , a fim de se verificar se o acidente ou a violência não tinha ocorrido no ano de 2001, porém nenhum caso foi observado. Também não se observou óbito ocorrido em 2001 cujo acidente ou violência houvesse ocorrido no ano anterior.

Foram considerados como acidentes ou violências os eventos classificáveis nos códigos do capítulo XX (causas externas de morbidade e de mortalidade) da Classificação Internacional de Doenças, décima revisão (CID-10). O trauma gerado pelo aciden- te ou violência foi classificado segundo os códigos do capítulo XIX dessa Classificação (lesões, envenenamentos e algumas outras conseqüências de causas externas) ${ }^{1}$.

Os dados de morbidade foram processados eletronicamente por meio do programa de domínio público Epi Info - versão $6.04 \mathrm{~d}^{17}$, excluindo-se os atendimentos duplicados referentes a um mesmo acidente ou violência. Os óbitos que ocorreram sem assistência foram incluídos com vistas a formar um único banco de dados de morbimortalidade.

Os menores de 15 anos vítimas de acidentes e violências foram distribuídos em três grupos de análise: os atendidos somente em pronto-socorro e que receberam alta, os internados não-fatais e os óbitos.

O presente estudo foi submetido ao Comitê de Ética em Pesquisa da Universidade Estadual de Londrina, sendo aprovado. Os dados foram coletados de outubro de 2002 a abril de 2003, por uma equipe de alunos do curso de Enfermagem da Universidade Estadual de Londrina, devidamente treinados e supervisionados.

\section{Resultados}

Foram estudados 8.854 menores de 15 anos vítimas de acidentes ou violências no ano de 2001 , sendo que $95,7 \%$ (8.472) foram atendidos no pronto-socorro e liberados. As internações não-fatais somaram $364(4,1 \%)$. Entre os 18 óbitos $(0,2 \%)$, sete $(38,9 \%)$ ocorreram no local do acidente ou da violência, quatro $(22,2 \%)$ morreram ao receber assistência no pronto-socorro e sete $(38,9 \%)$ chegaram a ser internados. A Figura 1 mostra a situação da população menor de 15 anos em cada um dos níveis de assistência após a ocorrência do acidente ou violência.

Estes resultados permitem estimar que, para cada óbito de menor de 15 anos por uma causa externa, aproximadamente 470 são atendidos exclusivamente em prontosocorro e cerca de 20 são internados.

Entre os internados, o tempo de internação variou de 1 a 37 dias, sendo que $47,6 \%$ ficaram internados um dia, $24,2 \%$ dois 


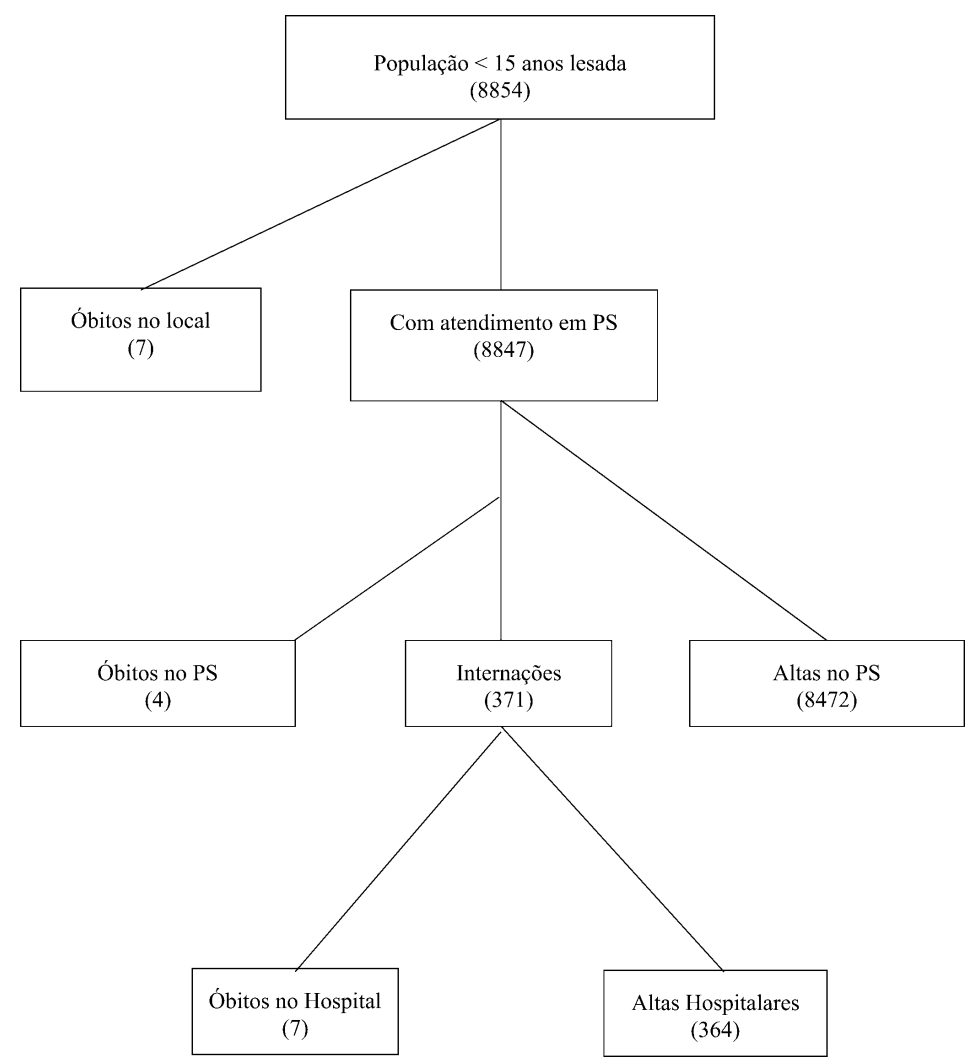

Figura 1 - Situação da população menor de 15 anos lesada em acidentes/violências, Londrina, 2001

Figure 1 - Situation of the population under 15 years of age injured in accidents/violence, Londrina, 2001

dias, $10,4 \%$ três dias e $6,9 \%$ quatro dias, totalizando $89,1 \%$ com internação de um a quatro dias. Outros $8,2 \%$ ficaram internados de 5 a 10 dias e $2,7 \%$ mais de 10 dias.

Entre as crianças e adolescentes que morreram, o tempo entre o acidente/violência e $\mathrm{o}$ falecimento variou de 0 a 11 dias, sendo que 11 casos $(61,1 \%)$ morreram menos de 24 horas após o evento, três casos $(16,7 \%)$ ocorreram de 24 a 48 horas e outros quatro casos $(22,0 \%)$ após quatro, cinco, oito e onze dias.

A Tabela 1 mostra que, entre as vítimas atendidas somente em pronto-socorro, com alta, houve predomínio de quedas $(33,9 \%)$, seguidas de eventos de intenção indeterminada ( $31,8 \%)$, acidentes causados por forças mecânicas inanimadas $(15,5 \%)$ especialmente por penetração de corpo estranho em orifício natural $(4,6 \%)$ ou através da pele $(3,2 \%)$ - e acidentes de transporte $(7,0 \%)$.

As quedas continuaram sendo as principais responsáveis pelas internações não-fatais $(32,4 \%)$ (Tabela 2$)$, seguidas dos acidentes de transporte $(19,5 \%)$, acidentes causados por forças mecânicas inanimadas $(15,7 \%)$ - sobretudo por penetração de corpo estranho em orifício natural $(4,1 \%)$ ou através da pele $(3,0 \%)$ - envenenamentos $(13,5 \%)$. As causas externas de intenção indeterminada reduziram-se, nesse nível de atenção, para apenas 5,5\%.

Acidentes de transporte $(44,4 \%)$, afogamento $(16,7 \%)$ e aspiração de conteúdo gástrico $(11,1 \%)$ foram as principais causas de óbito por causa externa nessa população (Tabela 3). Dos óbitos por afogamento, dois ocorreram em coleção de água natural e um em balde de água. Ainda outros tipos de aci- 
Tabela 1 - Distribuição dos menores de 15 anos atendidos em pronto-socorro, por causas externas (CID-10), Londrina, 2001.

Table 1 -Distribution of individuals under 15 years of age who received emergency care due to external causes (ICD-10), Londrina, 2001.

\begin{tabular}{lrr}
\hline Causas externas (subgrupos) & № & $\%$ \\
\hline Acidentes de Transporte & 590 & 7,0 \\
Quedas & 2872 & 33,9 \\
Forças mecânicas inanimadas & 1317 & 15,5 \\
$\quad$ Impacto causado por objeto lançado ou em queda & $(117)$ & $(1,4)$ \\
$\quad$ Impacto causado por equipamento esportivo & $(50)$ & $(0,6)$ \\
$\quad$ Impacto contra objetos & $(160)$ & $(1,9)$ \\
$\quad$ Apertado ou comprimido por ou entre objetos & $(79)$ & $(0,9)$ \\
Contato com material cortante & $(195)$ & $(2,3)$ \\
Queima de fogos de artifício & $(3)$ & $(0,0)$ \\
Penetração de corpo estranho em orifício natural & $(393)$ & $(4,6)$ \\
$\quad$ Penetração de corpo estranho através da pele & $(269)$ & $(3,2)$ \\
Forças mecânicas inanimadas não especificadas & $(51)$ & $(0,6)$ \\
Forças mecânicas animadas & 330 & 3,9 \\
$\quad$ Golpe infligido por outra pessoa & $(19)$ & $(0,2)$ \\
Colisão entre duas pessoas & $(16)$ & $(0,2)$ \\
Mordedura de rato & $(1)$ & $(0,0)$ \\
Mordedura ou golpe provocado por cão & $(199)$ & $(2,3)$ \\
$\quad$ Mordedura / golpe provocado por outros animais & $(17)$ & $(0,2)$ \\
Mordedura/picada inseto/artrópode não venenoso & $(63)$ & $(0,7)$ \\
Mordedura provocada por outros répteis & $(1)$ & $(0,0)$ \\
Contato com espinhos de plantas & $(4)$ & $(0,0)$ \\
Forças mecânicas animadas não especificadas & $(10)$ & $(0,1)$ \\
Riscos à respiração & 23 & 0,3 \\
Exposição à corrente elétrica & 5 & 0,0 \\
Exposição ao fogo & 21 & 0,2 \\
Exposição à substância quente & 132 & 1,6 \\
Contato com animais ou plantas venenosas & 187 & 2,2 \\
Exposição às forças da natureza & 5 & 0,0 \\
Envenenamento (intoxicação) & 235 & 2,8 \\
Excesso de esforço & 1 & 0,0 \\
Lesões Auto-Provocadas Intencionalmente & 9 & 0,1 \\
Agressões & 49 & 0,6 \\
Intenção indeterminada & 2691 & 31,8 \\
Complicação de assistência médica & 5 & 0,1 \\
\hline TOTAL & 8472 & 100,0 \\
\hline
\end{tabular}

dente/violência foram causas de óbito na população de estudo, como uma por agressão por arma de fogo, uma por exposição às forças da natureza (vítima de raio), uma por exposição à corrente elétrica (contato com fio elétrico de poste de iluminação ao empinar pipa), uma por queda de escada. Apenas um óbito $(5,5 \%)$ foi considerado evento de intenção indeterminada, cuja causa não foi possível de ser elucidada, mesmo após investigação realizada pelo Núcleo de Informação em Mortalidade da Prefeitura de Londrina.

A região do corpo mais afetada foi a da cabeça/pescoço, tanto entre as vítimas atendidas em pronto-socorro quanto entre as internadas e, especialmente, entre as que foram a óbito (Tabela 4). 
Tabela 2 - Distribuição das internações de menores de 15 anos (casos não fatais) segundo subgrupos de causas externas (CID-10), Londrina, 2001.

Table 2 -Distribution of hospitalizations of individuals under 15 years of age (non fatal cases) according to subgroups of external causes (ICD-10), Londrina, 2001.

\begin{tabular}{lrr}
\hline Causas externas (subgrupos) & $N$ & $\%$ \\
\hline Acidente de Transporte & 71 & 19,5 \\
Queda & 118 & 32,4 \\
Acidentes causados por forças mecânicas inanimadas & 57 & 15,7 \\
$\quad$ Impacto causado por objeto lançado ou em queda & $(11)$ & $(3,0)$ \\
Impacto causado por equipamento esportivo & $(2)$ & $(0,5)$ \\
Impacto contra objetos & $(7)$ & $(1,9)$ \\
Apertado ou comprimido por ou entre objetos & $(3)$ & $(0,8)$ \\
Contato com material cortante & $(6)$ & $(1,6)$ \\
Queima de fogos de artifício & $(1)$ & $(0,3)$ \\
Penetração de corpo estranho em orifício natural & $(15)$ & $(4,1)$ \\
Penetração de corpo estranho através da pele & $(11)$ & $(3,0)$ \\
Forças mecânicas inanimadas não especificadas & $(1)$ & $(0,3)$ \\
Acidentes causados por forças mecânicas animadas & 10 & 2,7 \\
$\quad$ Mordedura ou golpe provocado por cão & $(6)$ & $(1,6)$ \\
Mordedura / golpe provocado por outros animais & $(2)$ & $(0,6)$ \\
Mordedura/picada inseto/artrópode não venenoso & $(2)$ & $(0,6)$ \\
Afogamento & 4 & 1,1 \\
Risco à respiração & 1 & 0,3 \\
Exposição ao fogo & 5 & 1,4 \\
Exposição à substância quente & 18 & 4,9 \\
Contato com animais ou plantas venenosas & 2 & 0,5 \\
Envenenamento (intoxicação) & 49 & 13,5 \\
Lesão Auto-Provocada Intencionalmente & 5 & 1,4 \\
Agressão & 4 & 1,1 \\
Intenção indeterminada & 20 & 5,5 \\
\hline TOTAL & 364 & 100,0 \\
\hline
\end{tabular}

Tabela 3 - Distribuição dos óbitos de menores de 15 anos vítimas de causas externas segundo subgrupos de causas (CID-10), Londrina, 2001.

Table 3 -Distribution of deaths of individuals under 15 years of age who were victims of external causes according to subgroups of causes (ICD-10), Londrina, 2001.

\begin{tabular}{lrr}
\hline Causas externas (subgrupos) & № & $\%$ \\
\hline Acidente de Transporte & 8 & 44,4 \\
Queda & 1 & 5,5 \\
Afogamento & 3 & 16,7 \\
Risco à respiração (aspiração de conteúdo gástrico) & 2 & 11,1 \\
Exposição à corrente elétrica & 1 & 5,5 \\
Exposição às forças da natureza & 1 & 5,5 \\
Agressão & 1 & 5,5 \\
Intenção indeterminada & 1 & 5,5 \\
\hline TOTAL & 18 & $100,0^{*}$ \\
\hline
\end{tabular}

* Aproximado para 100,0\% 
Tabela 4 - Distribuição de lesões em menores de 15 anos vítimas de causas externas segundo a região corpórea afetada e o nível de atendimento, Londrina, 2001.

Table 4 -Distribution of injuries in individuals under 15 years of age who were victims of external causes according to the body region affected and level of care, Londrina, 2001.

\begin{tabular}{lrrrrrr}
\hline Região Corpórea & Atendimentos de & Pronto-Socorro & \multicolumn{2}{c}{ Internações } & \multicolumn{2}{c}{ Óbitos } \\
Afetada & № & $\%$ & № & $\%$ & № & $\%$ \\
\hline Cabeça e pescoço & 2944 & 34,7 & 136 & 37,4 & 9 & 50,0 \\
Membros superiores & 2099 & 24,8 & 51 & 14,0 & - & - \\
Membros inferiores & 1835 & 21,7 & 39 & 10,7 & - & - \\
Múltiplas regiões & 258 & 3,0 & 23 & 6,3 & 1 & 5,5 \\
Intoxicação/corpo estranho & 1109 & 13,1 & 87 & 23,9 & 8 & 44,5 \\
Região não especificada & 227 & 2,7 & 28 & 7,7 & - & - \\
\hline Total & 8472 & 100,0 & 364 & 100,0 & 18 & 100,0 \\
\hline
\end{tabular}

Entre os atendimentos de pronto-socorro, o tipo de lesão mais freqüente foi o traumatismo superficial, seguido dos ferimentos (Tabela 5). Entre os internados, traumatismos superficiais e fraturas predominaram. Já entre os que morreram, o traumatismo intracraniano foi o principal tipo de lesão.

\section{Discussão}

Conhecer as causas de atendimento segundo o nível de atenção é essencial, uma vez que as causas externas não-fatais diferem das fatais, indicando a necessidade de diferentes formas de atenção e de prevenção ${ }^{14}$. Além disto, conhecer o perfil da de-

Tabela 5 - Distribuição de menores de 15 anos vítimas de causas externas segundo o nível de atendimento e o tipo de lesão, Londrina, 2001.

Table 5 - Distribution of individuals under 15 years of age who were victims of external causes according to level of care and type of injury, Londrina, 2001

\begin{tabular}{lrrrrrr}
\hline Tipo de Lesão & Atendimentos & de & Pronto-Socorro & \multicolumn{2}{c}{ Internações } & \multicolumn{2}{c}{ Óbitos } \\
& No & $\%$ & № & $\%$ & № & \% \\
\hline Traumatismo superficial & 2787 & 32,9 & 80 & 22,0 & - & - \\
Ferimento & 2483 & 29,3 & 48 & 13,2 & - & - \\
Fratura & 673 & 7,9 & 71 & 19,5 & - & - \\
Luxação & 591 & 7,0 & 4 & 1,1 & - & - \\
Traumatismo de nervo & 13 & 0,1 & 2 & 0,5 & - & - \\
Traumatismo de vaso & 72 & 0,8 & 4 & 1,1 & - & - \\
Traumatismo de músculo/tendão & 5 & 0,1 & - & - & - & - \\
Traumatismo intracraniano & 11 & 0,1 & 13 & 3,6 & 9 & 50,0 \\
Lesão por esmagamento & 13 & 0,1 & 4 & 1,1 & - & - \\
Amputação traumática & 1 & 0,0 & 2 & 0,5 & - & - \\
Trauma ap. urinário/órgão pélvico & 2 & 0,0 & 4 & 1,1 & - & - \\
Penetração de corpo estranho & 410 & 4,8 & 15 & 4,1 & - & - \\
Queimadura & 172 & 2,0 & 26 & 7,1 & - & - \\
Intoxicação & 426 & 5,0 & 55 & 15,1 & - & - \\
Asfixia & - & - & - & - & 5 & 27,8 \\
Efeito raio / choque & 2 & 0,0 & 4 & 1,1 & 2 & 11,1 \\
Reação vacinal & 5 & 0,1 & - & - & - & - \\
Outro trauma não especificado & 806 & 9,5 & 32 & 8,8 & 2 & 11,1 \\
\hline Total & 8472 & $100,0^{*}$ & 364 & $100,0 *$ & 18 & 100,0 \\
\hline
\end{tabular}

* Aproximado para 100,0\% 
manda pode colaborar na reorganização dos serviços de saúde, não só hospitalares, mas, inclusive, dos serviços de atenção básica à saúde do sistema municipal.

Os achados do presente estudo evidenciam que grande parte da demanda hospitalar gerada por conseqüências de acidentes ou violências na cidade de Londrina, entre menores de quinze anos, é de baixa complexidade, devido à elevada proporção de atendimentos em pronto-socorro com alta subseqüente. Todavia, essa característica não deve ser menosprezada, considerando que parte desses atendimentos poderia ter sido evitada, por meio de uma série de medidas preventivas consideradas efetivas ${ }^{18}$, o que proporcionaria redução dos gastos hospitalares com esses eventos e das situações de estresse vividas pela criança e por sua família.

Por outro lado, estes resultados sugerem, também, que alguns procedimentos menos complexos de atenção a essas causas poderiam ser implementados em unidades ambulatoriais do Município, como, por exemplo, a realização de suturas para ferimentos mais simples (segunda principal lesão entre os atendimentos de pronto-socorro), desafogando os setores de emergência/urgência hospitalares. Em Londrina, o Serviço Municipal de Saúde conta com uma ampla rede de unidades básicas de saúde e um serviço de pronto-atendimento infantil com funcionamento 24 horas/dia, o que proporciona extensa cobertura ambulatorial da população infantil. Estudar as causas de atendimento no Município em relação às causas externas pode colaborar com o planejamento da assistência e a reestruturação dos serviços, a fim de tornar mais eficiente o gerenciamento dos recursos.

Quanto aos atendimentos de pronto-socorro, às internações e aos óbitos, os resultados da presente casuística são semelhantes aos observados em outros estudos, como o de Filócomo et al. ${ }^{13}$, que verificaram, em São Paulo, 95,7\% de crianças vítimas de acidentes atendidas em pronto-socorro, com uma proporção de internação igual a 4,0\%. Resultados similares também foram encontrados em Campinas, entre crianças meno- res de quatorze anos vítimas de acidentes, com $97,1 \%$ de atendimentos em pronto-socorro e um percentual de $0,1 \%$ de óbitos ${ }^{9}$.

Em relação à duração da hospitalização, Baracat et al. ${ }^{9}$ observaram uma mediana de oito dias para crianças menores de 15 anos vítimas de acidente atendidas em Campinas, e Harada et al. ${ }^{19}$ encontraram uma média de internação de até 10,2 dias entre menores de 12 anos vítimas de acidente e atendidas em um hospital municipal de São Paulo. Ainda que a presente casuística tenha revelado que a maioria $(89,1 \%)$ das vítimas de acidentes e violências foi internada por um período de um a quatro dias, é importante ressaltar que a prevenção destes eventos resultaria na redução de gastos hospitalares, além do desgaste emocional vivenciado pela criança e pela família durante a permanência hospitalar. Estima-se que, em média, os acidentes infantis causem de 10 a $30 \%$ de ocupação de leitos hospitalares ${ }^{20}$. Desta forma, torna-se urgente a participação dos profissionais de saúde, bem como de outras categorias que direta ou indiretamente lidam com crianças ou adolescentes, na elaboração e na atuação nos programas de prevenção, que devem envolver também a família, profissionais, associações comunitárias e sociedades em geral.

Os resultados também permitem afirmar que a queda, motivo principal de atendimento em pronto-socorro e de internações nãofatais no presente estudo, constitui causa freqüente de acidentes infantis, o que também foi evidenciado por outros pesquisadores no Brasil ${ }^{9,13,19}$ e nos Estados Unidos da América $^{14}$. Tais achados indicam a pertinência de estudos adicionais, que determinem as circunstâncias e os fatores contribuintes para esses eventos na infância, para cada faixa etária, e que orientem medidas efetivas de prevenção.

Comparando-se a freqüência percentual dos eventos de intenção indeterminada nos três grupos de análise, observa-se melhora de informação sobre a intencionalidade da causa externa à medida que o acidente ou violência é mais grave, passando de $31,8 \%$ nos atendimentos de pronto-socorro para $5,5 \%$ nas internações e óbitos. A falta de re- 
gistro completo nas fichas de atendimento de urgência/emergência daqueles casos impossibilitou uma classificação específica do acidente ou violência segundo a Classificação Internacional de Doenças (CID-10) ${ }^{1}$, sendo necessário investir na melhora da qualidade dos registros nesse nível de atendimento, de forma a contribuir para um melhor conhecimento epidemiológico dessas causas de traumas infantis.

Os óbitos por acidentes de transporte, afogamentos e por aspiração de conteúdo gástrico, principais causas de morte na presente investigação, denunciam a gravidade desses tipos de acidentes, indicando, como conduta adequada para prevenir essa mortalidade, ser necessário investir na prevenção da ocorrência desses acidentes.

Vários estudos têm apontado o acidente de transporte como importante causa de morbidade e mortalidade ${ }^{21-23}$, e no presente trabalho os resultados não foram divergentes. Nos Estados Unidos, Ballesteros et al. ${ }^{14}$ identificaram o acidente de transporte, especificamente entre ocupantes de automóvel, como a principal causa de óbito entre menores de 15 anos. No Brasil, estudos revelam que as crianças e os adolescentes são mais vulneráveis aos atropelamentos ${ }^{23,24}$, além dos acidentes com bicicleta ${ }^{24}$. Tornase necessário, portanto, desenvolver estratégias eficazes de policiamento e educação no trânsito, além da criação de espaços seguros para a recreação de crianças ${ }^{22}$.

Os afogamentos, em geral, apresentam alta letalidade. Em Campinas, também se destacaram devido à elevada proporção de óbitos no total de acidentados $(50,0 \%)^{9}$. Nos Estados Unidos, esse tipo de acidente foi identificado como a terceira causa de óbito em crianças abaixo de 15 anos $^{14}$. A alta letalidade entre os afogamentos denuncia a gravidade desse tipo de acidente e a necessidade de medidas preventivas. Proteção ao redor de piscinas, aparentemente, não evitaria todos estes acidentes, uma vez que as mortes, no presente estudo, deram-se por afogamento em água natural e em balde de água. É preciso, portanto, ampliar as ações preventivas. As escolas de natação privilegiam, em geral, o treinamento em piscinas, gerando falsa sensação de segurança em ambientes naturais, onde as crianças, por seu pequeno tamanho e baixo peso, associados ao medo e à adversidade do ambiente, não conseguem sair de uma situação de emergência. Nesse sentido, é preciso investir na educação e orientação precoce das crianças. O uso de bóias e a vigilância são indispensáveis. No ambiente doméstico, é preciso estar atento a baldes, bacias, tanques, banheiras e recipientes com água, pois alguns momentos de distração podem ser suficientes para um acidente fatal.

Já a aspiração não foi responsável por óbitos no estudo realizado em Campinas ${ }^{9}$, mas ocupou a quarta colocação de todos os acidentes, com $7,4 \%$ de freqüência. Harada et al. ${ }^{19}$ afirmam que, de cada 10 episódios de aspiração, nove são perfeitamente evitáveis. Os óbitos por aspiração de conteúdo gástrico, observados neste estudo, indicam a necessidade de se ampliar e reforçar as orientações sobre sua prevenção durante o prénatal e nas atividades de puericultura.

A presença de agressões, inclusive com um óbito por projétil de arma de fogo, denuncia o aumento da violência nos últimos anos em cidades de médio porte no Brasil ${ }^{25}$ e aponta para a necessidade de legislação e intervenções mais eficazes, em especial as que coíbam a entrada de adolescentes no tráfico de drogas.

Os traumatismos cranianos, considerados de maior risco para conseqüências mais graves do trauma, também foram identificados por Baracat et al..$^{9}$ como lesão freqüente entre crianças acidentadas, especialmente entre menores de um ano que sofrem quedas. Do total de pessoas internadas por traumatismo craniano pelo sistema público de saúde no Brasil, cerca de $20 \%$ são crianças menores de 10 anos $^{26}$. A importante freqüência de trauma craniano pode estar relacionada ao estágio de desenvolvimento das crianças de menor idade, pois estas são incapazes de proteger a região cefálica em caso de quedas como, por exemplo, do berço ou de outras mobílias, da própria altura ao começar a andar e de impactos causados por ou contra 
objetos $^{14}$. Já os traumatismos de membros, em geral predominam nas crianças maiores ${ }^{9}$, provavelmente porque, além de já terem desenvolvido o reflexo de proteção da cabeça, uma das atividades características desta fase é a prática de esportes, como andar de bicicleta ou patins, jogos de bola, entre outros, nas quais há grande exposição a traumas dos membros inferiores e superiores.

Resultados similares aos do presente estudo são encontrados em alguns trabalhos que trazem os traumatismos superficiais, os ferimentos e as fraturas como sendo as lesões mais freqüentes nas crianças em virtude de acidentes. Lyons et al. ${ }^{27}$, por exemplo, encontraram um coeficiente de fraturas em crianças do País de Gales igual a 63,1 por 1.000 crianças. Segundo os autores, a cada ano, os ferimentos decorrentes de acidentes são responsáveis pelo atendimento de um quinto da população infantil do Reino Unido e da América do Norte. Em estudo nacional, Filócomo et al. ${ }^{13}$ apontam os ferimentos como o segundo tipo de lesão mais freqüente entre crianças de 0 a 14 anos, precedidos pela contusão.

Os resultados do presente estudo revelam que os acidentes infantis diferem-se de acordo com o nível de atenção, exigindo ações diferenciadas. A grande demanda de atendimentos por trauma superficial em pronto-socorro evidencia a necessidade de reestruturação dos serviços de saúde para que as unidades básicas tenham recursos humanos e materiais para o atendimento de crianças e adolescentes vítimas de acidente ou violência, reduzindo a demanda para os serviços terciários e os custos hospitalares. É preciso que as equipes de saúde da atenção primária sejam treinadas e que se implante rotinas ou protocolos padronizados para o tratamento adequado das lesões resultantes dos acidentes e violências infantis.

Os resultados apontam, ainda, para a necessidade urgente da adoção de medidas preventivas, embasadas na educação, na legislação e na fiscalização, visando reduzir os gastos com a atenção a estes eventos e, sobretudo, o sofrimento vivido pela família e pela criança.

A alta freqüência de eventos de intenção indeterminada nos atendimentos de pronto-socorro torna premente a implantação de protocolos de atendimento às vítimas de causas externas nesse nível de atenção, o que poderia contribuir para a obtenção de um melhor conhecimento epidemiológico destas causas de morbi-mortalidade.

\section{Referências}

1. Organização Mundial de Saúde. Classificação Estatística Internacional de Doenças e Problemas Relacionados à Saúde (CID-10). Centro Colaborador da OMS para a Classificação de Doenças em Português. 10a revisão. 8⿳⺈ ed. São Paulo: Editora da Universidade de São Paulo (EDUSP); 2000.

2. Blank D. Prevenção e controle de injúrias físicas: saímos ou não do século 20? J Pediatr 2002; 78: 84-6.

3. Mello Jorge MHP. Acidentes e violências no Brasil - à guisa de conclusão. Rev Saúde Pública 1997; 31 (4 Supl); 51-4.

4. Mello Jorge MHP, Gotlieb SLD, Laurenti R. A saúde no Brasil: análise do período 1996 a 1999. Brasília: Organização Pan Americana da Saúde; 2001.

5. Rodriguez JG, Brown ST. Childhood injuries in the United States: a strategy for reduction of childhood injuries in Massachusetts. Am J Dis Child 1990; 144: 625-6.
6. Souza LJEX, Barroso MGT. Acidente doméstico em crianças: abordagem conceitual. Acta Paulista de Enfermagem (São Paulo) 1999; 70-7.

7. Del Ciampo LA, Ricco RG. Acidentes na infância. Rev Pediatr 1996; 18: 193-7.

8. Souza LJEX, Barroso MGT. Revisão bibliográfica sobre acidentes com crianças. Rev Esc Enfermagem USP 1999; 33: 107-12.

9. Baracat ECE, Paraschin K, Nogueira RJN, Reis MC, Fraga AMA, Sperotto G. Acidentes com crianças e sua evolução na região de Campinas, SP. J Pediatr 2000; 76(5): 368-74.

10. Scherer EA, Sherer ZAP. A criança maltratada: uma revisão da literatura. Rev Latino-am Enf 2000; 8: 22-9.

11. Fonseca SS, Victora CG, Halpern R, Barros AJD, Lima $\mathrm{RC}$, Barros FC et al. Fatores de risco para injúrias acidentais em pré-escolares. J Pediatr 2002; 78: 97-104. 
12. Barros MDA, Ximenes R, Lima MLC. Mortalidade por causas externas em crianças e adolescentes: tendências de 1979 a 1995. Rev Saúde Pública 2001; 35: 142-9.

13. Filócomo FRF, Harada MJS, Silva CV, Pedreira MLG. Estudo dos acidentes na infância em um prontosoccoro pediátrico. Rev Latino-am Enf 2002; 10(1): 41-7.

14. Ballesteros MF, Schieber RA, Gilchrist J, Holmgreen P, Annest JL. Differential ranking of causes of fatal versus non-fatal injuries among US children. Inj Prev 2003; 9: 173-6.

15. Mello Jorge MHP, Gotlieb SLD, Laurenti R. Crianças, adolescentes e jovens do Brasil no fim do século XX. In: Westphal MF, org. Violência e criança. São Paulo: EDUSP; 2002. p. 47-72.

16. DATASUS. DATASUS [dados da Internet]. População residente. [acessado em 15 Out 2002]. Disponível em: $<$ http://tabnet.datasus.gov.br/cgi/tabcgi.exe?ibge/ cnv/poppr.def $>$.

17. Dean AG, Dean JA, Coulombier D, Brendel KA, Smith DC, Burten AH et al. Epi Info, version 6: a word processing, database and statistics program for epidemiology on microcomputers. Centers for Disease Control and Prevention, Atlanta, USA; 1995.

18. Towner E, Dowswell T, Jarvis S. Updating the evidence. A systematic review of what works in preventing childhood unintentional injuries: Part I. Inj Prev 2001; 7: 161-4.

19. Harada MJCS, Botta MLG, Kobata CM, Szauter IH, Dutra G, Dias EC. Epidemiologia em crianças hospitalizadas por acidentes. Folha Med 2000; 119: 437.
20. Blank D. Conceitos básicos e aspectos preventivos gerais. In: Comitê de acidentes na infância. Manual de acidentes na infância e adolescência. São Paulo: Sociedade Brasileira de Pediatria; 1994.

21. Scalassara MB, Souza RKT, Soares DFPP. Características da mortalidade por acidente de trânsito em localidade da região Sul do Brasil. Rev Saúde Pública 1998; 32: 125-32.

22. Andrade SM, Mello Jorge MHP. Acidentes de transporte terrestre em município da Região Sul do Brasil. Rev Saúde Pública 2001; 35: 318-20.

23. Deslandes SF, Silva CMFP. Análise da morbidade hospitalar por acidentes de trânsito em hospitais públicos do Rio de Janeiro, Brasil. Rev Saúde Pública 2000; 4: 367-72.

24. Andrade SM, Mello Jorge MHP. Características das vítimas por acidentes de transporte terrestre em município da Região Sul do Brasil. Rev Saúde Pública 2000; 34: 149-56.

25. Lima MLC, Souza ER, Ximenes R, Albuquerque MFPM, Bitoun J, Barros MDA. Evolução de homicídios por área geográfica em Pernambuco entre 1980 a 1998. Rev Saúde Pública 2002; 36: 462-9.

26. Koizumi MS, Mello Jorge MHP, Nóbrega LRB, Waters C. Crianças internadas por traumatismo crânioencefálico no Brasil, 1998: causas e prevenção. Inf Epidemiol SUS 2001; 10: 93-101.

27. Lyons RA, Delahunty AM, Kraus D, Heaven M, Cabe MM, Allen H, Nash P. Children's fractures: a population based study. Inj Prev 1999; 5: 129-32.

recebido em: 17/12/04 versão final reapresentada em: 23/05/05 aprovado em: 03/06/05 